\title{
Impacto da pureza dos filtros de alumínio no valor de Camada Semi-Redutora em radiologia convencional e mamografia
}

\author{
N. W. Lima ${ }^{a}$; G. Hoff ${ }^{b}$ \\ ${ }^{a}$ Faculdade de Física, PUCRS 90619-900, CEP,Porto Alegre, RS, Brasil \\ ${ }^{b}$ Bolsista da CAPES - Projeto No 161/2012 \\ lima.nathan@gmail.com
}

\begin{abstract}
Half Value Layer (HVL) test is an important technical information in conventional radiology once it is the only test which allows us to quantify beam quality in a complete way. This work promotes a preliminary analysis of the im-pact of aluminum alloy composition in the determination of HVL value taking into account a realistic geometry. Deterministic calculations and Monte Carlo Method were applied in order to make the proposed analysis. Six alu-minum alloys (99\% pure) commercialized and pure aluminum were used as comparative basis for both methods. Experimental data were collected in conventional radiology, using tungsten target and aluminum filter for two peak tensions (66 $\mathrm{kVp}, 81 \mathrm{kVp}$ ), and in mammography, using three target filter combinations( molybdenum-molybdenum, molybdenum-rhodium, tungstenrhodium) for three peak tensions (25 kVp, $30 \mathrm{kVp}$ and $35 \mathrm{kVp}$ ). Spectra used in the deterministic calculations were taken from Report No 78 of Institute of Physics and Engineering in Medicine, 1997. Results show that Mammography is more sensitive than conventional $X$-ray equipment to impu-rities in aluminum alloys (up to $34.65 \%$ variation for $W$ - $R h$ combination with $30 \mathrm{kVp}$ )
\end{abstract}

KEY WORDS: Radiology, mammography, dosimetry. 


\section{INTRODUÇÃO}

O teste de Camada Semi-Redutora (CSR) é realizado no controle da qualidade de aparelhos de raios $\mathrm{X}$ destinados à radiologia diagnóstica (radiologia convencional, mamografia e fluoroscopia), conforme previsto na Portaria 453 (ANVISA, 1998). Associado a outros testes, ele pode auxiliar no rastreamento de problemas no tubo gerador de radiação $\mathrm{X}$ antes que esses problemas se tornem perceptíveis e diminuam a qualidade diagnóstica das imagens (Nagel; 1988).

O teste de CSR, conforme descrito por Lopes (2013) e Villafana (1990) consiste em medir a exposição $(\mathrm{X})$ em geometria de irradiação fixa (distância entre ponto focal real e a superfície do detector é mantida constante), realizada de tal forma que a coleta de retroespalhamento do bucky ou da mesa de exames seja minimizada. São realizadas incidências acrescentando filtros, de um material conhecido, entre o tubo de raios X e o detector até que o valor medido seja inferior à metade do valor inicial (Xinicial) coletada sem material filtrante entre o ponto focal real e o detector). Determina-se, então, por interpolação logarítmica qual é a espessura de material necessária para reduzir Xinicial à metade, sendo esse valor chamado de Camada Semi-Redutora.

O teste é feito, no Brasil, geralmente, utilizando uma câmara de ionização e filtros de alumínio de alto grau de pureza. Podem-se encontrar, no mercado, diferentes filtros de alumínio com pureza entre 99\% e 99,99\%. Publicações da década de 90 mostraram que para mamografia analógica, $1 \%$ de impureza nos filtros de alumínio poderia implicar em erros de até $10 \%$ dez por cento no teste de CSR (WAGNER et al, 1990). Por outro lado, não foi encontrado na literatura nenhum artigo que mostre essa relação em mamografia e radiologia digital, uma vez que existem mudanças significativas em termos de características do tubo de raios $\mathrm{X}$ com o avanço tecnológico e, portanto, no espectro emitido pelo tubo. Tinha-se por hipótese que haveria diferença nos resultados desse trabalho em comparação ao trabalho da década de 1990.

\section{MATERIAIS E MÉTODOS}

A metodologia empregada é descrita sob três aspectos: arranjo experimental, modelo determinístico, e simulação computacional.

a) Arranjo Experimental

As medidas experimentais da exposição $(\mathrm{X})$ foram utilizadas como base comparativa de validação dos métodos matemáticos estudados. Para a realização das medidas de $\mathrm{X}$ foi utilizada uma câmara de ionização para radiologia e mamografia, do conjunto TNT 12000 da Fluke, devidamente calibrada.

Os aparelhos geradores de radiação X utilizados foram um Multix Top da Siemens, para avaliação na área da radiologia convencional, e um MammomatInspiration da Siemens, para avaliações na área da mamgrafia. Ambos os equipamentos apresentaram os 
resultados de todos os testes de controle da qualidade dentro dos limites estabelecidos pela legislação brasileira (ANVISA, 1998). Os filtros de alumínio (Al) utilizados pertenciam ao conjunto TNT 1200 da Fluke, com pureza de 99\% garantida.

Na radiologia convencional, as medidas de exposição foram realizadas para distância fixa entre o ponto focal e o detector de $84,5 \mathrm{~cm}$. Esse aparelho de raios $\mathrm{X}$ apresenta trilha de tungstênio e filtração total equivalente a 2,5 mm de Al. Foram realizadas medidas em tensões de pico de $66 \mathrm{kV}$ e de $81 \mathrm{kV}$.

Na mamografia, as medidas de exposição foram realizadas para distância fixa entre o ponto focal e o detector de $41,0 \mathrm{~cm}$, considerando as combinações trilha-filtro: molibdênio-molibdênio (Mo-30Mo), sendo a espessura do filtro adicional de $30 \mu \mathrm{m}$, para tensões de pico de $25 \mathrm{kV}$ e $30 \mathrm{kV}$, molibdênio-ródio (Mo-25Rh), sendo a espessura do filtro adicional de $25 \mu \mathrm{m}$, para tensões de pico de $25 \mathrm{kV}$ e $30 \mathrm{kV}$ e tungstênio-ródio (W-50Rh), sendo a espessura do filtro adicional de $50 \mu \mathrm{m}$, para tensões de pico de $30 \mathrm{kV}$ e $35 \mathrm{kV}$. Foram utilizados dois modelos matemáticos sendo um modelo de atenuação exponencial para o transporte da radiação e outro baseado no Método de Monte Carlo. Para ambos os estudos matemáticos foram considerados espectros do Catalogue of DiagnosticXraySpectraandother Data- Report (CRANLEY et al, 1997) para descrição do espectro do feixe primário de radiação. Os espectros primários simulados apresentaram características idênticas às dos espectros utilizados no arranjo experimental.

Para realizar a comparação proposta por este projeto, entre as diferentes composições de filtros de alto grau de pureza, foram obtidas as composições nominais de seis ligas de alumínio comercializadas mundialmente para a realização do teste de CSR. As composições avaliadas para ambos os métodos de cálculo utilizados estão descritas na Tabela 1.

Tabela 1. Composição nominal das ligas de alumínio utilizadas para realização dos cálculos determinísticos e as simulações por método de Monte Carlo.

\begin{tabular}{lcccccc}
\hline Elemento & Liga 1 & Liga 2 & Liga 3 & Liga 4 & Liga 5 & Liga 6 \\
\hline Al & $99,00 \%$ & $99,00 \%$ & $99,00 \%$ & $99,00 \%$ & $99,00 \%$ & $99,00 \%$ \\
Mn & $0,05 \%$ & $0,05 \%$ & $0,05 \%$ & - & - & - \\
Si & $0,95 \%$ & - & $0,475 \%$ & - & $0,9 \%$ & $0,45 \%$ \\
Fe & - & $0,95 \%$ & $0,475 \%$ & $0,90 \%$ & - & $0,45 \%$ \\
Zn & - & - & - & $0,10 \%$ & $0,10 \%$ & $0,1 \%$ \\
\hline
\end{tabular}

Para determinação do valor de CSR foi utilizada a ferramenta Solver do software livre Gnumeric (Free Software Foundantion, 2015) além de dois métodos de cálculo da CSR: um determinando o valor pela interpolação $\ln$-ln dos dados de espessura e exposição e International Joint Conference RADIO 2014 Gramado, RS, Brazil, August 26-29, 2014 SOCIEDADE BRASILEIRA DE PROTEÇÃO RADIOLÓGICA - SBPR 
outro determinando a CSR pela equação de ajuste polinomial aos dados. Essa verificação foi realizada para observar diferenças significativas que poderiam ser ocasionadas pelo uso de métodos diferentes. As maiores diferenças foram verificadas entre os métodos de interpolação ln-ln e interpolação polinomial, mas sempre foram diferenças inferiores ou da ordem de grandeza das flutuações do método. Optou-se, então utilizar o software Gnumeric (ferramenta Solver), pois os valores calculados por ele ficaram intermediários aos extremos.

b) Modelo Determinístico

Para implementação do modelo determinístico, os coeficientes de atenuação linear total dos diferentes materiais envolvidos no problema foram considerados com a finalidade de se determinar matematicamente a CSR para as diferentes ligas de alumínio. Na descrição da atenuação do feixe, levou-se em conta um feixe monodirecional sendo atenuado pela filtração total de aparelho de raios X, pelo ar e pelos filtros adicionais de alumínio, utilizados na realização do teste de CSR. Os coeficientes de atenuação linear utilizados foram determinados pela base de dados XCOM do National Institute of Standartsand Technology (BERGER, 2014) - que calcula o coeficiente de atenuação do material composto fazendo uma ponderação em massa dos coeficientes de atenuação dos diferentes componentes. Primeiramente, calculou-se a exposição gerada, por fótons de energia i, pelo feixe incidente no ponto imediatamente posterior ao filtro adicional $\left(X_{0 i}\right)$ :

$$
X_{0 i}=X_{0 i}^{\prime} e^{-\sum \mu_{i} j x_{j}}
$$

onde $X_{0 i}^{\prime}$ é a exposição gerada pelos fótons de energia i no ponto de emissão, $\mu_{i j}$ é o coeficiente de atenuação para os fótons de energia $i$ causada pelo meio $j$ (existem três meios atenuadores - o filtro do colimador, a janela do tubo e o ar) e $x_{\mathrm{j}}$ é a espessura do meio atenuador $j$. Para se ter a exposição total causada por um feixe policromático, devese, então somar as exposições resultantes de cada componente energética. Deve-se lembrar que $\mathrm{X}$ se relaciona com a fluência de energia pela seguinte relação:

$$
X=\sum \varphi_{i}\left(\frac{\mu_{\text {tr }}}{\rho}\right)_{i}^{a r}\left(\frac{e}{w}\right)
$$

onde $\varphi_{i}$ é a fluência de energia para os fótons de energia i, $\left(\frac{\mu_{t r}}{\rho}\right)_{i}^{a r}$ é o coeficiente de transmissão de energia do ar para a energia i, e representa a carga do elétron e W é a energia média necessária para formar um par de íons no ar, o valor de W/e médio costuma ser contabilizado como 33,97 J/C (ATTIX, 1986). Por fim, CSR que representa espessura de filtro que fornece a redução da exposição pela metade, pode ser expressa pela equação 3.

$$
d_{1 / 2}=\ln 2 / \mu
$$

Para determinação do valor de CSR foi utilizada a ferramenta Solver do software livre Gnumeric (FREE SOFTWARE FOUNDATION, 2015). Considerando os dados gerados 
pelo modelo determinístico, nenhuma variação foi associada aos mesmos, sendo este dado de variação preenchido com NA, que significa não aplicável.

c) Simulação Computacional

A simulação em Monte Carlo foi realizada utilizando o conjunto de ferramentas computacionais denominado Geant4 (ALLISON et al, 2006; AGOSTINELLI et al, 2003). Para definir o mundo virtual, utilizou-se uma geometria similar àquela utilizada no arranjo experimental, respeitando as características do feixe (alvo, filtração), simulando a fonte como pontual e o detector como uma câmara de ionização de 15 centímetros cúbicos de volume sensível.

A implementação de uma fonte pontual é aceitável visto que o tamanho do ponto focal é consideravelmente menor do que as distâncias empregadas no arranjo experimental (no caso da mamografia, a razão entre essas dimensões chega a um para mil, na radiologia convencional essa razão é ainda maior).

A descrição do transporte de radiação nos meios materiais, foi selecionada a biblioteca de seç̧ão choque e modelos de baixa energia “Low Energy Livermore Model”. Foram definidos valores de corte de $0,1 \mathrm{~mm}$ para todas as partículas que atingem o volume sensível da câmara de ionização e 0,1 mm para fótons e 1,0 m para elétrons para o resto do mundo. $\mathrm{O}$ volume do detector foi definido considerando uma câmara de ionização free in air com 15 cc de volume sensível. A fonte foi definida como pontual irradiando fótons homogeneamente numa área circular de raio 2,08 cm na entrada da superfície da câmara de ionização. Foram usadas $10^{8}$ histórias para cada run para chegar nos resultados com a estatística adequada. As variações registradas nos dados de CSR devem-se somente às flutuações estatísticas provenientes do método de Monte Carlo não sendo computadas as incertezas epistemológicas envolvidas.

\section{RESULTADOS E DISCUSSÕES}

Os resultados serão apresentados na seguinte ordem: validação (nas tabelas 2 e 3) e comparação entre filtros pelos resultados dos métodos matemáticos aplicado (tabelas de 4 a 7). Pode-se observar na Tabela 2 que as variações entre o cálculo determinístico (CD) e os dados experimentais de $15,4 \%$ e $22,8 \%$ e variações entre o método de Monte Carlo (MC) e os dados experimentais de -20,1\% e -11,7\% para as energias de $66 \mathrm{kVp}$ e $81 \mathrm{kVp}$. $\mathrm{O}$ único caso em que a variação entre $\mathrm{MC}$ e os dados experimentais foi maior que para a comparação com os CD, em todo o estudo foi o caso de radiologia convencional a 66 $\mathrm{kVp}$. 
Tabela 2. Comparação entre os valores da CSR, em mm, definido pelos dados experimentais e pelos modelos matemáticos para a área da radiologia convencional.

\begin{tabular}{cccccc}
\hline & & \multicolumn{2}{c}{66 kVp } & \multicolumn{2}{c}{81 kVp } \\
\cline { 3 - 6 } & & CSR (mm) & Variação \% & CSR (mm) & Variação \% \\
\hline \multirow{2}{*}{ Experimental } & Interpolação ln-ln & 2,65 & 0,21 & 3,24 & 0,10 \\
\cline { 2 - 6 } & Equação de ajuste & 2,63 & 0,20 & 3,20 & 0,12 \\
\hline \multirow{2}{*}{ Cálculo Determinístico } & 3,11 & NA & 3,93 & \multirow{2}{*}{ NA } \\
\hline \multicolumn{2}{c}{ Monte Carlo } & 2,10 & 0,13 & 2,84 & 0,14 \\
\hline
\end{tabular}

A Tabela 3 mostra os valores de CSR para as diferentes condições de contorno aplicada a área da mamografia. Observa-se, que em todos os casos da mamografia, as diferenças entre os métodos de cálculo e os dados experimentais foram menores entre MC e experimento. Nas tabelas a seguir, a sigla utilizada descreve respectivamente o material do alvo, a espessura do filtro, o material do filtro e a tensão de pico utilizada. Por exemplo, Mo-30Mo@25 representa a combinação de alvo de Molibdênio com filtro de 25 micrometros de Molibdênio e tensão de pico de 25 kV.

Tabela 3. Comparação entre os valores da CSR, em mm, definido pelos dados experimentais e pelos modelos matemáticos para a área da mamografia, considerando as combinações molibdênio-molibdênio para 25 kVp (Mo-30Mo@25) e 30kVp (Mo-30Mo@30), molibdênio-ródio para 25kVp (Mo-25Rh@25) e 30 kVp

(Mo-25Rh@30) e tungstênio-ródio para 30kVp (W-50Rh@30) e 35 kVp (W50Rh@35)

\begin{tabular}{|c|c|c|c|c|c|}
\hline & & \multicolumn{2}{|c|}{ Mo-30Mo@25 } & \multicolumn{2}{|c|}{ Mo-30Mo@30 } \\
\hline & & $\mathrm{CSR}(\mathrm{mm})$ & Variação \% & CSR (mm) & Variação \% \\
\hline \multirow[t]{2}{*}{ Experimental } & Interpolação ln-ln & 0,28 & 3,8 & 0,34 & 2,1 \\
\hline & Equação de ajuste & 0,27 & 3,8 & 0,32 & 2,1 \\
\hline \multicolumn{2}{|c|}{ Método Determinístico } & 0,37 & NA & 0,43 & NA \\
\hline \multirow{2}{*}{\multicolumn{2}{|c|}{ Monte Carlo }} & 0,25 & 0,66 & 0,29 & 0,25 \\
\hline & & \multicolumn{2}{|c|}{ Mo-25Rh@25 } & \multicolumn{2}{|c|}{ Mo-25Rh@30 } \\
\hline
\end{tabular}




\begin{tabular}{|c|c|c|c|c|c|}
\hline & & CSR (mm) & Variação \% & CSR (mm) & Variação \% \\
\hline \multirow{2}{*}{ Experimental } & Interpolação ln-ln & 0,34 & 1,1 & 0,39 & 0,7 \\
\hline & Equação de ajuste & 0,34 & 1,1 & 0,39 & 0,7 \\
\hline \multicolumn{2}{|c|}{ Método Determinístico } & 0,43 & NA & 0,49 & NA \\
\hline \multicolumn{2}{|c|}{ Monte Carlo } & 0,34 & 1,2 & 0,38 & 0,4 \\
\hline & & \multicolumn{2}{|c|}{ W-50Rh@30 } & \multicolumn{2}{|c|}{ W-50Rh@35 } \\
\hline & & CSR (mm) & Variação \% & $\mathrm{CSR}(\mathrm{mm})$ & Variação \% \\
\hline \multirow[t]{2}{*}{ Experimental } & Interpolação ln-ln & 0,47 & 1,1 & 0,53 & 0,80 \\
\hline & Equação de ajuste & 0,47 & 1,1 & 0,53 & 0,80 \\
\hline \multicolumn{2}{|c|}{ Método Determinístico } & 0,64 & NA & 0,72 & NA \\
\hline \multicolumn{2}{|c|}{ Monte Carlo } & 0,39 & 6,0 & 0,45 & 3,0 \\
\hline
\end{tabular}

É importante observar que o CD sempre superestimou os dados de CSR, e os MC subestimou. As diferenças foram, com exceção de um caso, sempre inferiores entre MC e os dados experimentais.

Os resultados estão organizados em quatro tabelas, nas quais são apresentados os valores de CSR obtidos pelo CD e pelo MC para as diferentes combinações alvo-filtro e tensões de pico.

Maiores variações foram verificadas para os alvos de $\mathrm{W}$, isso provavelmente acontece devido ao fato de alterações no espectro tabelado para este alvo. Estudos adicionais devem ser realizados para confirmar esta hipótese.

Tabela 4. Resultados de CSR para combinação molibdênio-molibdênio para 25 kVp (Mo-30Mo@25) e 30 kVp (Mo-30Mo@30).

\begin{tabular}{cccccc}
\cline { 2 - 5 } & \multicolumn{2}{c}{ Mo-30Mo@25 } & \multicolumn{2}{c}{ Mo-30Mo@30 } \\
\cline { 2 - 5 } & CSR (mm) & Variação & CSR (mm) & Variação \\
\hline Al & 0,37 & NA & 0,43 & NA \\
Liga 01 & 0,36 & NA & 0,43 & NA \\
CD Liga 02 & 0,34 & NA & 0,40 & NA \\
Liga 03 & 0,35 & NA & 0,42 & NA \\
Liga 04 & 0,34 & NA & 0,40 & NA
\end{tabular}




\begin{tabular}{|c|c|c|c|c|c|}
\hline & \multirow{2}{*}{$\begin{array}{l}\text { Liga } 05 \\
\text { Liga } 06\end{array}$} & \multirow{2}{*}{$\begin{array}{l}0,36 \\
0,35\end{array}$} & \multirow{2}{*}{$\begin{array}{l}\text { NA } \\
\text { NA }\end{array}$} & \multirow{2}{*}{$\begin{array}{l}0,42 \\
0,41\end{array}$} & \multirow{2}{*}{$\begin{array}{l}\text { NA } \\
\text { NA }\end{array}$} \\
\hline & & & & & \\
\hline & $\mathrm{Al}$ & 0,25 & $0,66 \%$ & 0,30 & $0,25 \%$ \\
\hline & Liga 01 & 0,24 & $0,74 \%$ & 0,30 & $0,25 \%$ \\
\hline & Liga 02 & 0,22 & $0,60 \%$ & 0,27 & $0,27 \%$ \\
\hline \multirow[t]{4}{*}{$\mathrm{MC}$} & Liga 03 & 0,24 & $0,70 \%$ & 0,29 & $0,27 \%$ \\
\hline & Liga 04 & 0,24 & $0,77 \%$ & 0,28 & $0,24 \%$ \\
\hline & Liga 05 & 0,22 & $0,74 \%$ & 0,30 & 0,30\% \\
\hline & Liga 06 & 0,23 & $0,55 \%$ & 0,29 & $0,32 \%$ \\
\hline
\end{tabular}

Pelos valores de CSR apresentados na Tabela 4, para $25 \mathrm{kVp}$ com a combinação Mo30Mo, a variação encontrada pelo CD foi 8,5\% em relação média dos valores calculados e pelo MC, a variação foi de $12,8 \%$. Para $30 \mathrm{kVp}$, com a combinação Mo-30Mo, a variação encontrada pelo CD foi de 7,21\% e, pelo MC, a variação foi de 10,34\%.

Tabela 5. Resultados de CSR para combinação molibdênio-ródio para 25kVp (Mo-25Rh@25) e 30 kVp (Mo-25Rh@30).

\begin{tabular}{cccccc} 
& & \multicolumn{2}{c}{ Mo-25Rh@25 } & \multicolumn{2}{c}{ Mo-25Rh@30 } \\
\cline { 2 - 5 } & & CSR (mm) & Variação & CSR (mm) & Variação \\
\hline Ll & 0,43 & NA & 0,49 & NA \\
Liga 01 & 0,43 & NA & 0,49 & NA \\
Liga 02 & 0,41 & NA & 0,47 & NA \\
CD & Liga 03 & 0,42 & NA & 0,48 & NA \\
& Liga 04 & 0,41 & NA & 0,47 & NA \\
& Liga 05 & 0,43 & NA & 0,49 & NA \\
Liga 06 & 0,42 & NA & 0,48 & NA \\
\cline { 2 - 5 } MC & Al & 0,34 & 1,29\% & 0,38 & $0,42 \%$
\end{tabular}




\begin{tabular}{crrrr} 
Liga 01 & 0,34 & $1,16 \%$ & 0,36 & $0,44 \%$ \\
Liga 02 & 0,32 & $1,13 \%$ & 0,34 & $0,52 \%$ \\
Liga 03 & 0,33 & $1,14 \%$ & 0,40 & $0,42 \%$ \\
Liga 04 & 0,31 & $1,07 \%$ & 0,38 & $0,39 \%$ \\
Liga 05 & 0,33 & $1,05 \%$ & 0,42 & $0,33 \%$ \\
Liga 06 & 0,30 & $1,44 \%$ & 0,40 & $0,36 \%$ \\
\hline
\end{tabular}

Pelos valores de CSR apresentados na Tabela 5, para 25 kVp com a combinação Mo-Rh, a variação verificada para CD foi de 4,74\% e para MC, 12,33\%. Para $30 \mathrm{kVp}$, com a combinação Mo-Rh, a variação calculada para CD foi de 4,15\% e para MC, 10,44\%.

Tabela 6. Resultados de CSR para combinação tungstênio-ródio para $30 \mathrm{kVp}$ (W50Rh@30)e 35 kVp (W-50Rh@35).

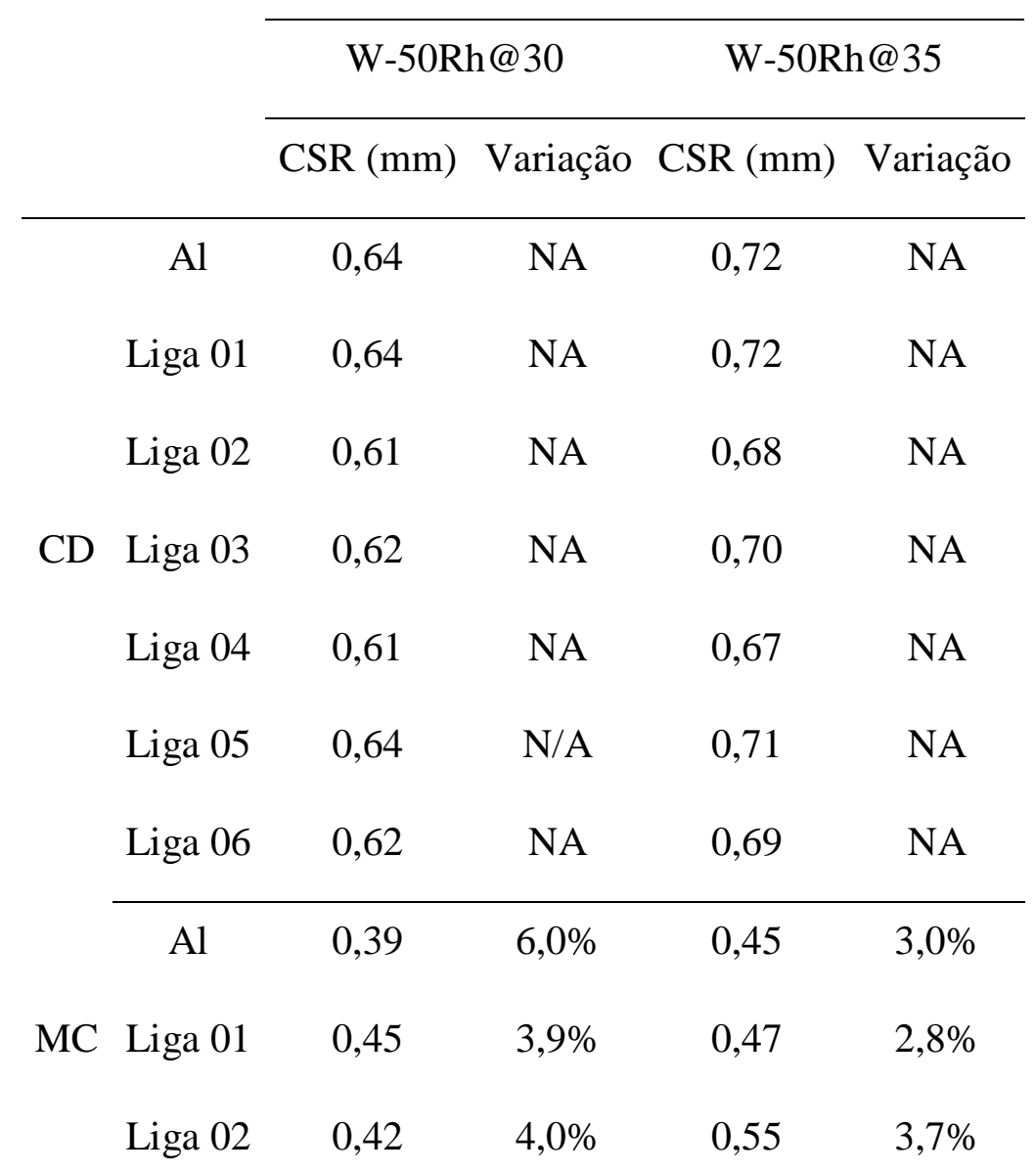




\begin{tabular}{rrrrr} 
Liga 03 & 0,54 & $4,7 \%$ & 0,46 & $3,7 \%$ \\
Liga 04 & 0,42 & $3,3 \%$ & 0,46 & $2,7 \%$ \\
Liga 05 & 0,42 & $3,7 \%$ & 0,46 & $2,8 \%$ \\
Liga 06 & 0,39 & $4,7 \%$ & 0,50 & $3,5 \%$ \\
\hline
\end{tabular}

Pelos valores de CSR apresentados na Tabela 6, para $30 \mathrm{kVp}$, com a combinação W-Rh, a variação calculada para CD foi de 4,79\% e para MC, 34,65\%.

Os dados daTabela 6, para $35 \mathrm{kVp}$ na combinação W-Rh, a variação encontrada pelo CD foi de 5,72\% e pelo MC, 20,89\%.

Tabela 7. Resultados de CSR para combinação tungstênio-alumínio para 66 kVp (WAl@66) e 81 kVp (W-Al@81).

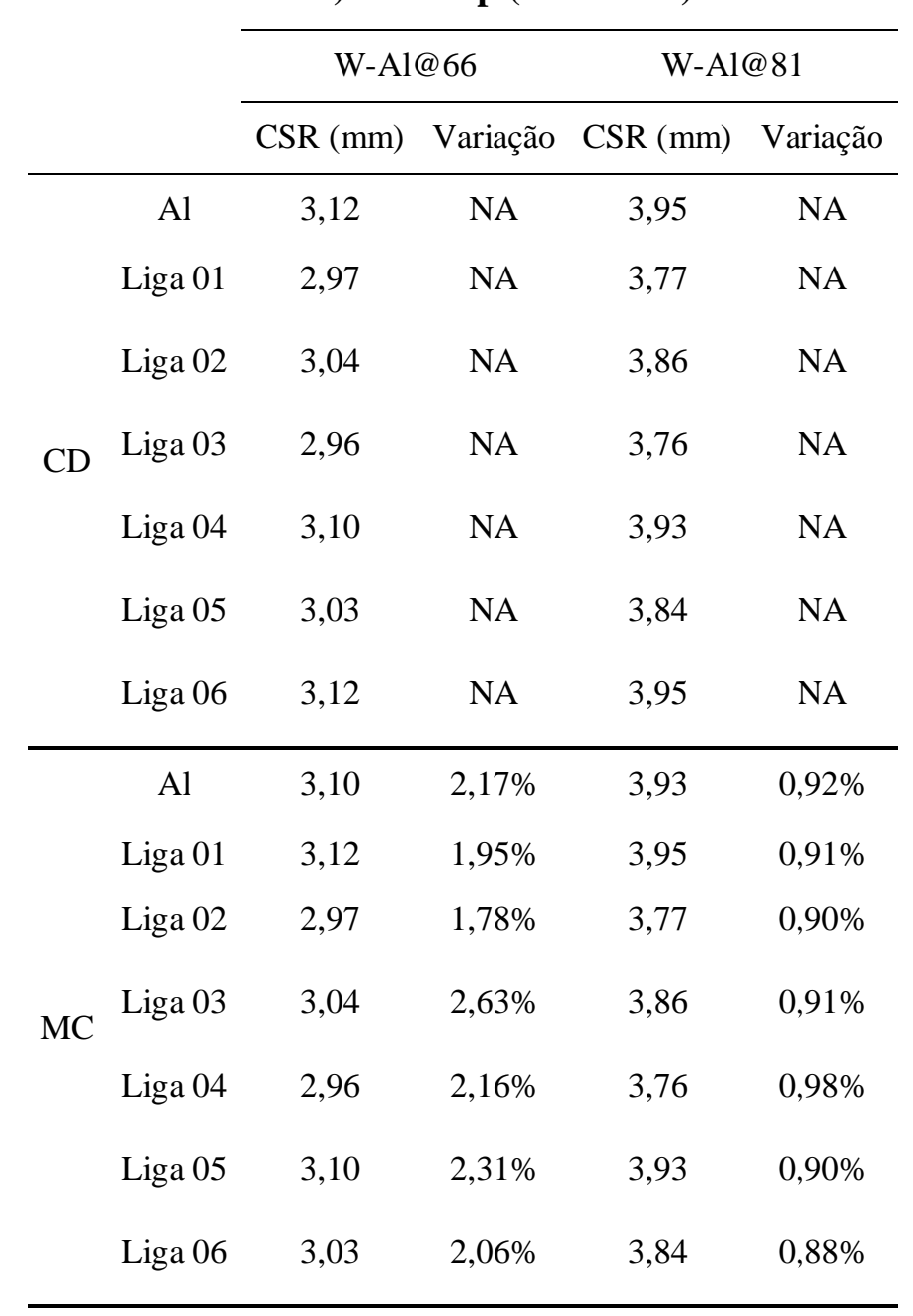


Pelos valores de CSR apresentados na Tabela 7, na radiologia convencional, onde se tem alvo de tungstênio e filtração de alumínio, para $66 \mathrm{kVp}$, a variação determinada para CD foi de 5,16\% e por MC, 5,25\%. Para $81 \mathrm{kVp}$, a variação calculada para CD foi de 4,91\% e para MC foi de $4,65 \%$.

\section{CONCLUSÕES}

Apesar de diferenças significativas terem sido verificadas nos resultados para alvos de W (que podem ter sido ocasionadas por diferenças entre os espectros experimentais e tabelados), o método de MC apresentou menores diferenças entre os dados calculados e experimentais, mostrando melhor concordância com este método de cálculo. Com base nessa informação, levou-se em consideração os dados calculados pelo método de MC como base para refutar a afirmação de que em mamografia $1 \%$ de impureza no filtro resulta em $10 \%$ de erro na estimativa da CSR, pois verificou-se variações geralmente superiores a $10 \%$ na mamografia. Na radiologia convencional, conforme o esperado, esse erro foi menor, mesmo assim ele ficou na ordem de $5 \%$.

Percebe-se que o método de MC é mais sensível à variação da composição das ligas em comparação com o CD. Isso se deve ao fato de que o modelo determinístico empregado avalia apenas o efeito de atenuação da radiação ao passar pelos diferentes meios materiais, ou seja, não leva em conta a radiação espalhada na direção da câmara de ionização, enquanto que a simulação em MC, mais realista, leva em consideração os diferentes fenômenos de espalhamento possíveis de acordo com as diferentes seções de choque dos materiais estudados. Essa característica, associada a melhor concordância entre os dados experimentais e o método de MC, tornam os valores de CSR determinados por este método mais confiável, sendo o que melhor representa as flutuações entre as diferentes composições de filtro.

Além disso, é evidente que as variações encontradas na mamografia são superiores às calculadas para a radiologia convencional, chegando ao valor máximo de 34,65\% para a combinação tungstênio-ródio com $30 \mathrm{kVp}$. Isso pode ser explicado, porque, em baixas energias, as seções de choque do Efeito Fotoelétrico são proporcionais ao número atômico na quarta potência7, o que significa que variações no número atômico influenciam de forma significativa a probabilidade de atenuação da radiação.

\section{AGRADECIMENTOS}

Os autores agradecem ao Hospital de Clínicas de Porto Alegre, especialmente aos Físicos Alexandre Bacelar e RochelleLykawka que possibilitaram a realização da coleta de dados experimental. Agradecemos também à Dra. Elaine EvaniStreck pelas contribuições e sugestões significativas técnicas e teóricas. 


\section{REFERÊNCIAS}

AGOSTINELLI, S. et al. GEANT 4 - a simulation toolkit. Nuclear Instruments and Methods in Physics Research Section A: Accelerators, Spectrometers, Detectors and Associated Equipment, v 506, Issue 3, p.250-303. 2003.

ALLISON, J. et al. Geant Developments and Applications. IEEE Transactions on Nuclear Science, v.53, p. 270-278, 2006.

ANVISA - Agência Nacional de Vigilância Sanitária - Ministério da Saúde - Secretaria de Vigilância Sanitária. Diretrizes de ProteçãoRadiológicaemRadiodiagnóstico Medico e odontológico. ANVISA Portaria 453.1998.

ATTIX, F. H. Introduction to Radiological Physics and Radiation Dosimetry. New York: J. Wiley;1986.

CRANLEY, K. et al. Catalogue of Diagnosis X ray Spectra and Other Data. Report 78 The Institute of Physics and Engineering in Medicine - Report 78. 1997

FREE SOFTWARE FOUNDATION. Gnumeric. Disponível em: http://www.gnumeric.org/. GNU GPL Protect. Últimoacesso: 03 de Fevereiro de 2015.

LOPES, G. A. et al. Determinação de Camada Semi-Redutora e Deci-Redutora para Barita com blindagem contra radiação X em Proteção Radiológica. International Nuclear Atlantic Conference . Recife, 2013.

NAGEL, H. D. Limitations in the determination of the total filtration of x-ray tube assemblies. Phys Med Biol, v.33. p. 271-28, 1988. 
BERGER, M. J. at al XCOM: Photon Cross Sections Database. Disponívelem http://physics.nist.gov/PhysRefData/Xcom/html/xcom1.html. The NationalInstituteof Standards and Technology (NIST) isanagencyofthe U.S. DepartmentofCommerce. Último Acesso: 09 de setembro de 12014. .

VILLAFANA, T. Generators, x-ray tubes, and exposure geometry in mammography. Radiographics, v.10, p.539-54,1990.

WAGNER, L. K.; ARCHER, B. R.; CERRA, F. On the measurement of half-value layer in film-screen mammography. Med Phys, v.17, p.989-97, 1990. 